\title{
A Synthetic Aperture Radar Image Model of Ship on Sea
}

\author{
Tong Wang ${ }^{1}$, Chuang-ming Tong ${ }^{1}$, Qian $\mathrm{Gao}^{2}, \mathrm{Xi}-\mathrm{min} \mathrm{Li}^{1}$ \\ ${ }^{1}$ Air Force Engineering University, Changle road No. 1, Xi'an, China \\ ${ }^{2}$ Air Force Communication NCO Academy, Dalian, China
}

\begin{abstract}
Double scattering between the ship and sea surface is an important characteristic for ship detection. Based on geometrical optics (GO), a modified GO-GO solution is presented to evaluate the double scattering from sea and the tilt facets of ship at high frequency. Direct scattering contributions from ship and sea are evaluated by using physical optics (PO). To get a synthetic aperture radar (SAR) image, double bounce and shadow effects on range dimension of SAR image are considered. The results show the relationship between the azimuth angle and the scattering power. The research on SAR image of ship-sea and results about double scattering between ship and sea have a great application value in ship detection from SAR image.
\end{abstract}

\section{INTRODUCTION}

Many ships have huge size and their two sides will form dihedral or approximate dihedral. In this situation, multiple scattering will dominate the backscattering field. This phenomenon is very useful for ship detection in SAR image. Sea surface is generally rough because of sea wind. At high frequency region, $\mathrm{GO}$ and $\mathrm{PO}$ are often used to evaluate the scattering from ground or sea. Both of the models are very appealing for rough surface scattering simulation due to their ease of implementation and easily identifying important underlying physical processes (Franceschetti, 2002; Masona, 2014; Ferro, 2008). Combining with the ray tracing theory, double scattering model is proposed for dealing with the SAR imaging problem between canonical target and rough surface. GO-GO, GO-PO is the simplest models for the double scattering model for their closed form and less parameters (Franceschetti, 2002). GO-GO and GO-PO are used based on the roughness of surface respectively. However, both of the models are first proposed to cope with the SAR imaging of urban scene in which the target is the vertical building (Franceschetti, 2003; Ferro, 2008). For ocean scene, the laterals of ship are not vertical to the mean sea, so the application of the simplest double scattering models in the ocean scene is need to be considered (Iervolino, 2016). Especially the situation in which the lateral sides of ship are not vertical to the mean sea.

\section{SCATTERING MODEL}

\subsection{DOUBLE SCATTERING}

In order to get simple scattering field expression, we assume the probability density function of sea surface follow Gaussian function which will be used in the following $\mathrm{GO}$ and $\mathrm{PO}$ models. PO solution approximation uses tangent plane approximation to obtain the expressions for surface fields. Zero-order PO solution can be given

$$
\begin{gathered}
\gamma_{a b}^{\mathrm{PO}}=\frac{\left|\bar{k}_{d}\right|^{4}}{4\left|\hat{k}_{i} \times \hat{k}_{s}\right|^{4} k_{d z}^{2}} f_{a b} \exp \left(-k_{d z}^{2} \sigma^{2}\right) \sum_{m=1}^{\infty} \frac{\left(k_{d z}^{2} \sigma^{2}\right)^{m}}{m ! m} \\
l^{2} \exp \left[-\frac{\left(k_{d x}^{2}+k_{d y}^{2}\right) l^{2}}{4 m}\right]
\end{gathered}
$$

where subscripts $a$ and $b$ refer to the scatter and incident wave polarizations. $\bar{k}_{d}$ is the difference wave vector of the incident wave unite vector and scattered wave unite vector $\left(\bar{k}_{d}=\hat{k}_{i}-\hat{k}_{s}\right) . \sigma$ is the standard deviation and $l$ is the correlation length of rough surface. Function $f_{\mathrm{ab}}$ depends on the Fresnel coefficients of the rough surface evaluated at stationary phase point and polarization information of incident wave and scattered wave, which can be defined as 


$$
\begin{aligned}
& f_{v v}=\left|\left(\hat{h}_{s} \cdot \hat{k}_{i}\right)\left(\hat{h}_{i} \cdot \hat{k}_{s}\right) R_{h}+\left(\hat{v}_{s} \cdot \hat{k}_{i}\right)\left(\hat{v}_{i} \cdot \hat{k}_{s}\right) R_{v}\right|^{2} \\
& f_{h v}=\left|\left(\hat{v}_{s} \cdot \hat{k}_{i}\right)\left(\hat{h}_{i} \cdot \hat{k}_{s}\right) R_{h}-\left(\hat{h}_{s} \cdot \hat{k}_{i}\right)\left(\hat{v}_{i} \cdot \hat{k}_{s}\right) R_{v}\right|^{2} \\
& f_{v h}=\left|\left(\hat{h}_{s} \cdot \hat{k}_{i}\right)\left(\hat{v}_{i} \cdot \hat{k}_{s}\right) R_{h}-\left(\hat{v}_{s} \cdot \hat{k}_{i}\right)\left(\hat{h}_{i} \cdot \hat{k}_{s}\right) R_{v}\right|^{2} \\
& f_{h h}=\left|\left(\hat{v}_{s} \cdot \hat{k}_{i}\right)\left(\hat{v}_{i} \cdot \hat{k}_{s}\right) R_{h}+\left(\hat{h}_{s} \cdot \hat{k}_{i}\right)\left(\hat{h}_{i} \cdot \hat{k}_{s}\right) R_{v}\right|^{2}
\end{aligned}
$$

When the incident wave frequency is very high $(k \sigma \gg$ 1 , wavenumber $k_{0}$ towards infinity or equivalently as well wavelength is much smaller than the radius of curvature of rough surface), GO solution can be obtained by using the method of stationary phase. GO model means that the stationary points which will specularly reflect the incident wave into observation direction contribute the most portions to the received field in observation direction. GO solution can be written in a simple form as

$$
\begin{aligned}
\gamma_{a b}^{\mathrm{GO}}\left(\hat{k}_{s}, \hat{k}_{i}\right)= & \frac{S\left|\bar{k}_{d}\right|^{4}}{\cos \theta_{i}\left|\hat{k}_{i} \times \hat{k}_{s}\right|^{4} k_{d z}^{4}} \frac{1}{2 h^{2}\left|C^{\prime \prime}(0)\right|} \\
& \exp \left[-\frac{k_{d x}^{2}+k_{d y}^{2}}{2 k_{d z}^{2} h^{2}\left|C^{\prime \prime}(0)\right|}\right] f_{a b}
\end{aligned}
$$

Where

$$
\left|C^{\prime \prime}(0)\right|=2 h^{2} / l^{2}
$$

$\theta_{i}$ is the angle between the incident direction and normal of the surface. $S$ is the illuminated area.

In the double scattering model, we regard every facet as smooth plan. In order to approximate complex geometry targets, triangle facet is introduced. The geometry of ship-sea model is defined as Fig. 1.

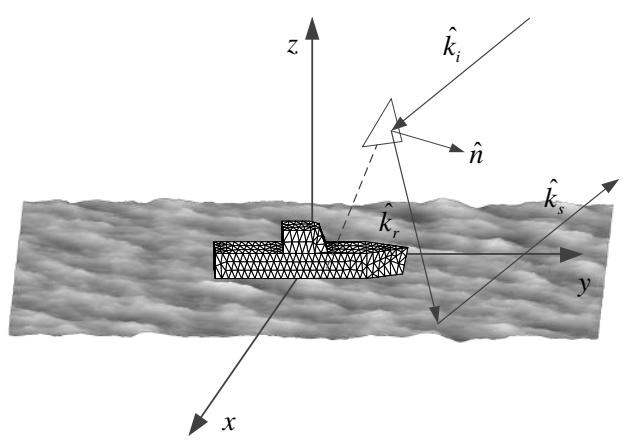

Fig. 1 Geometry of ship-sea model

In Fig. 1, we can see that some parts of the ship form dihedrals with sea surface, so multiple scattering between the ship and sea arises in this situation. Due to more than triple scattering is very small compared with the double scattering and direct scattering, only double scattering contributions are considered in the multiple scattering. Simple ship geometry model is regarded perfect metallic conductor which is composed of a series of triangle facets. When vector $\hat{n}$ i.e. the normal of one triangle facet is normal to $\mathrm{z}$ axis, then a perfectly dihedral is formed. For a perfect dihedral, analytic solutions in closed form based on GO-GO and GO-PO can be achieved. If $\hat{n}$ is not normal to $\mathrm{z}$ axis, closed form solution cannot be obtained but double scattering may be also strong depending on the angle of facet inclination. In this situation equation (1) and (3) can be applied.

\subsection{SAR IMAGING}

In SAR image simulation, the shadow effect needs to be considered. Z-buffer technology is adopted to estimate the illuminated facets of ship and the shadow area of sea surface shielded by the ship.

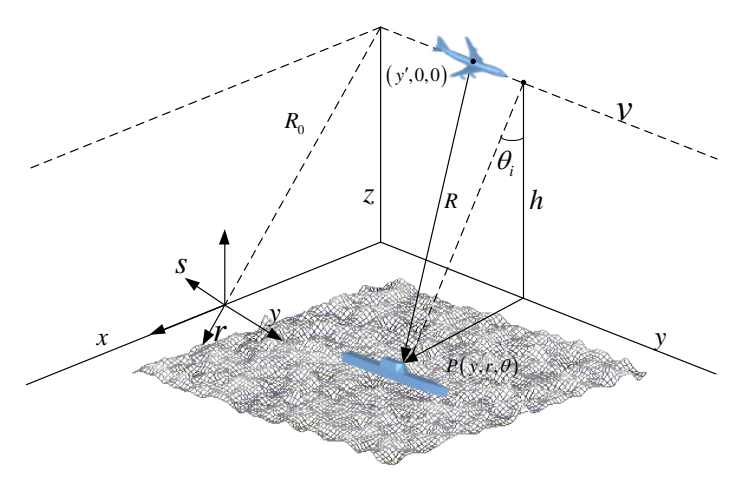

Fig. 2 SAR system geometry of ship-sea

Once the amplitudes the signals are determined based above considerations, raw signal can be simulated.

$$
\begin{aligned}
& h_{\text {strip }}\left(x^{\prime}, y^{\prime}\right)=\iint \gamma(y, r) \exp \left[-j \frac{4 \pi}{\lambda} \frac{\Delta f}{f c \tau}\left(r^{\prime}-r-\Delta R\right)^{2}\right] \\
& \omega^{2}\left(\frac{y^{\prime}-y}{X}\right) \exp \left(-j \frac{4 \pi}{\lambda} \Delta R\right) \operatorname{rect}\left[\frac{2\left(r^{\prime}-r-\Delta R\right)}{c \tau}\right] d y d r
\end{aligned}
$$

where $\gamma(y, r)$ including the phase factor $\exp (-\mathrm{i}(4 \pi)$ $\lambda)$ ) is the reflectivity of a small facet, which may be on the sea surface or ship. $R$ is the distance between radar platform and interested one point $P(y, r, \theta) . R_{0}$ is the minimum distance between the fight path and the central point of the scene. $\Delta f$ is bandwidth of radar transmitting wave. $c$ is the light velocity and $\tau$ is pulse duration. $X=\lambda R_{0} / L$ is the azimuth width of radar antenna illuminates. $\Delta R=R-r=\sqrt{r^{2}+\left(y^{\prime}-y\right)^{2}}$. we simply defined antenna gain $\omega(\cdot)$ is the same in the illuminated area. $r^{\prime}$ is the result of multiplying between pulse delay time and $c / 2$. Superposition of directly returns from all the illuminated facets constitutes the directly part of SAR raw signal. For double scattering contributions, $\gamma(y, r)$ is replaced by double scattering value and the path is the route of double bounce between ship and sea.

\section{SIMULATION AND RESULTS}

The geometry of the ship in our simulation is shown in Fig. 3. The lateral sides are tilted compared with tanker and cargo ships. Many naval vessels adopt this shape so 
that they can move fast. The angle is 9.5 degrees between lateral surface of ship and sea surface.

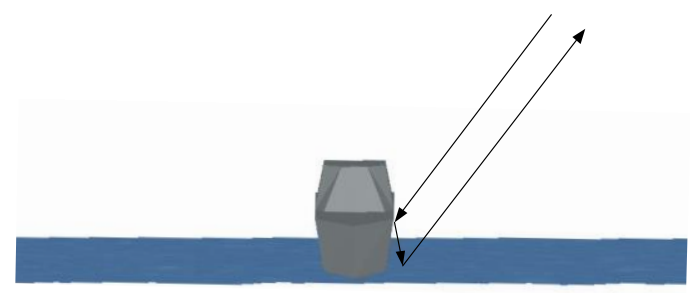

Fig.3 Geometry of ship-sea

In our simulation, radar works at $\mathrm{X}$ band, $\mathrm{GO}-\mathrm{GO}$ is more proper. Based on GO-GO model, the double reflection of the ships can be evaluated. The contribution of direct scattering from ship can be evaluated by the PO. RCS of every facet and the path of directly and double reflection are obtain, then the SAR raw signal are determined. Parameters used in the SAR signal simulation are shown as list.

Table 1. The parameters of SAR imaging

\begin{tabular}{|l|l|l|l|l|l|}
\hline$f(\mathrm{GHz})$ & $\theta_{i}$ & $\tau(\mathrm{um})$ & $\begin{array}{l}\Delta f \\
(\mathrm{MHz})\end{array}$ & $v(\mathrm{~m} / \mathrm{s})$ & $L(\mathrm{~m})$ \\
\hline 10 & $\pi / 3$ & 2 & 300 & 300 & 1.0 \\
\hline
\end{tabular}

$v$ is the velocity of flight. Firstly, we assume the azimuth angle is 0 degree which radar wave incident direction is vertical to the ship long axis. Sea wind is $5 \mathrm{~m} / \mathrm{s}$, relative permittivity of sea is $55-\mathrm{j} 25$.

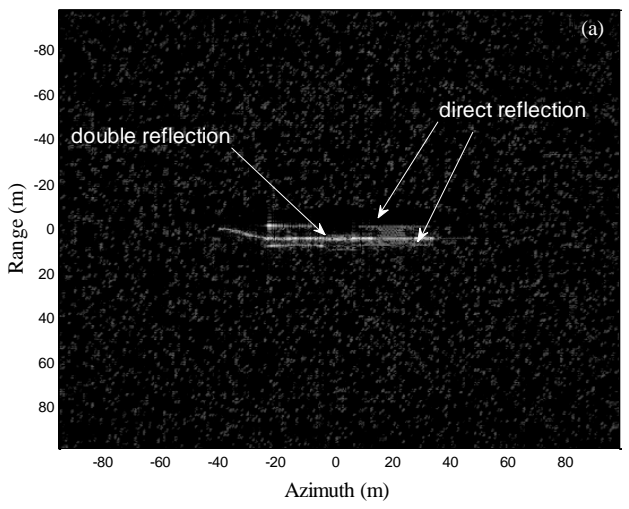

Fig. 4 SAR images of ship-sea

Fig. 4 illustrates the SAR image of ship on the sea. The incident angle is $20^{\circ}$. Fig. 4 clearly shows the double reflection effect on the SAR image. Double reflection behaves as a bright line in the SAR image, and brighter than directly reflection. Because ship is on the sea surface, double reflection line in the image is close to the directly reflection line of ship's illuminated side. (The location of double reflection is between the direct reflections of two sides of ship.) Directly reflection from bow is weakly, however the double reflection is apparent in SAR image while is also weaker than the double reflection from the vertical side. That is because bow line is not vertical to the incident direction, scattering power is weaker than the lateral of ship. Besides, the impact of clutter is very distinct as the result of small incident angle. We can find that the double scattering feature is easier to be recognized from the strong clutter.
Incident angle is changed into $60^{\circ}$. Changing the incident azimuth angle, the SAR images are showed as in Fig. 5.
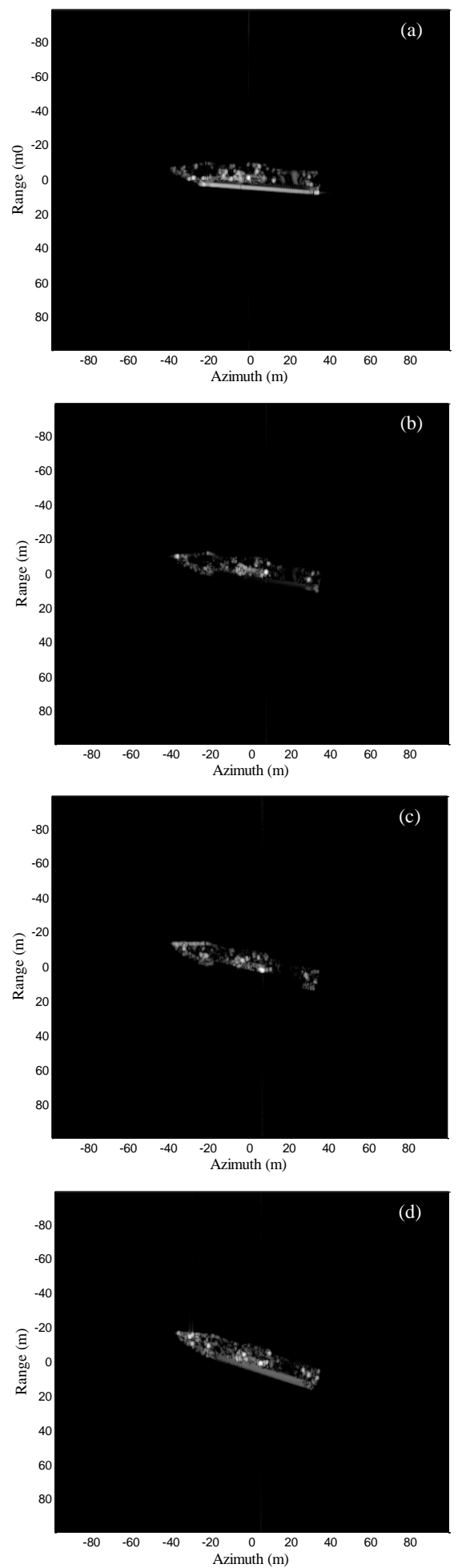

Fig. 5 SAR image of different azimuth angles without sea reflection

In Fig. 5(a) we can see double reflection is also strong compare to directly reflection. The clutter is very weak as the result of the increasing of incident angle. Fig. 5(b)-(c) show that with the variation of azimuth angle, double reflection becomes weaker. Correspondingly, directly reflection which previously is overwhelmed by double reflection contribution appears gradually in SAR image. 
In Fig. 5(d), we can see double reflection almost disappears and directly reflection perfectly shows the outline of ship.

\section{CONCLUSIONS}

Facet modified GO-GO and GO-PO models have been proposed to be applied into SAR imaging. The models evaluate the double scattering between tilt facet and sea surface simply and efficiently. At high frequency, GOGO is adopted. Scattering model of direct reflection, double reflection and shadow effects have been discussed. Finally, SAR images of ship on the sea are obtained. From SAR images, we find that with the azimuth angle increasing, double scattering becomes weaker. The phenomenon in image is agreement with physical interpretation. Double scattering is an important characteristic of ship on sea, so the research in this paper has great application value in ship detection in SAR image.

\section{REFERENCES}

1. Franceschetti G., Iodice A. and Riccio D., 2002. A canonical problem in electromagnetic backscattering from buildings. IEEE Trans. Geosci. Remote Sen., vol. 40(8), p. 1787-1801.

2. Franceschetti G., Iodice A., Riccio D. and Ruello G., 2003. SAR raw signal simulation for urban structures. IEEE Trans. Geosci. Remote Sens., 41(9), p. 1986-1995.

3. Masona D. C., Giustarini L, Pintado J. G. and. Cloke H. L, 2014. Detection of flooded urban areas in high resolution Synthetic Aperture Radar images using double scattering. Int. J. Appl. Earth Obs. Geoinf., 28(1), pp. 150-159,

4. Ferro A., Brunner D., Bruzzone L. and Lemoine G., 2011. On the relationship between double bounce and the orientation of buildings in VHR SAR images. IEEE Trans. Geosci. Remote Sens. Lett., 8(4), p. 612-616.

5. Iervolino P., Guida R. and Whittaker P., 2016. A model for the backscattering from a canonical ship in SAR imagery. IEEE Trans. Geosci. Remote Sens., 9(3), p. 1163-1175. 